\title{
Clinical Application and Exploration on Steps Progressively Corrections and Gradual Shortening Combined with Total Spinal Osteotomy for Angular Kyphosis-Basic Research and Clinical Application
}

\author{
Ming Lu', Xiaoping Wang1, Dongyun Ren'1, Huasong Ma ${ }^{2 *}$ \\ ${ }^{1}$ Department of Orthopedics, the 306 Hospital of Chinese PLA, Beijing, China \\ ${ }^{2}$ Department of Orthopedics, Military Center for Spine Surgery, the 306 Hospital of Military Chinese PLA, \\ Beijing, China \\ Email: *mahuasong@vip.163.com
}

Received 28 December 2015; accepted 25 January 2016; published 28 January 2016

Copyright (C) 2016 by authors and Scientific Research Publishing Inc.

This work is licensed under the Creative Commons Attribution International License (CC BY). http://creativecommons.org/licenses/by/4.0/

\section{(c) (i) Open Access}

\begin{abstract}
Introduction: This study is designed to explore the biomechanical principles of posterior bilateral pedicle screw on steps progressively and tight closure on every step for severe angular kyphosis and the indication conditions and clinical pathways of above new technique. Materials and Methods: A total of 90 patients have severe angular kyphosis, 37 males and 53 females, with an average age of 47 years. All patients were treated with posterior bilateral pedicle screw and step tight closure for gradual shortening and orthopedic technology. Results: Average intraoperative blood loss was $2089 \mathrm{ml}$. Average operation time was 326 minutes. Kyphotic angle changed from $90.1^{\circ}$ averagely preoperatively to $41.6^{\circ}$ averagely postoperatively with an improvement rate of $65 \%$. The distance from $C_{7}$ plumb line to posterior upper edge of the $S_{1}$ vertebral body was averagely 5.2 $\mathrm{mm}$ postoperatively, with a correction rate of $73 \%$. Neurological complications occurred in 2 cases, accounting for $6 \%$. Non-neurological complications occurred in 2 cases, accounting for $6 \%$. Anatomical parameters were significantly improved postoperatively $(P<0.05)$. All patients were averagely followed up for 28 months. Segments undergoing osteotomy achieved bone fusion, without loss of orthopedic angle. Conclusions: The orthopedic technology of bilateral pedicle screw and step tight closure designed by human physiology and biomechanical principles can protect spinal cord cells from injury to the utmost. To choose an appropriate osteotomy plane during pre-sur-
\end{abstract}

${ }^{*}$ Corresponding author.

How to cite this paper: Lu, M., Wang, X.P., Ren, D.Y. and Ma, H.S. (2016) Clinical Application and Exploration on Steps Progressively Corrections and Gradual Shortening Combined with Total Spinal Osteotomy for Angular Kyphosis-Basic Research and Clinical Application. Surgical Science, 7, 39-47. http://dx.doi.org/10.4236/ss.2016.71005 
gical planning can make the repair results closer to the normal function of the spine. Intraoperative nerve root protection, full release and bone graft fusion are effective safeguards to ensure kyphosis correction and to avoid spine lateral offset.

\section{Keywords}

\section{Angular Kyphosis, Total Spinal Osteotomy, Biomechanical Analysis, Clinical Outcome}

\section{Introduction}

The feature of spinal kyphotic deformity is that adjacent vertebral bodies are wedge-shaped and aggravate angular deformity of kyphosis apex [1]. Excessive flexion distraction of the spinal cord is further aggravated, and the highest point of tension is at the apex of the kyphosis [1]. Studies on spinal vessels of the angular kyphosis segments in childhood patients demonstrated that venous plexus on spinal pia mater of upper top vertebra had been expanded, but no venous plexus was found below the top vertebra. In some segments of the anterior spinal artery, spinal vessels connect each other by communicating branches. However, vascular spasm destroyed the decompensated state of spinal cord blood supply [2]. For patients whose spine growth is over, the aggravation of neurological symptoms is directly associated with the destructive blood supply of spinal vessels. Oppression of spinal cord and dural sac by malformation will lead to long-standing spinal cord compression syndrome.

With continuous modification and improvement of modern science and technique, studies concerning osteotomy therapy for angular kyphosis are gradually increasing. With orthopedic method gradually increased, more people pay attention to minimally invasive surgical techniques in orthopedic process and more stringent operating process. Thus, science, safety and effectiveness of surgical techniques gradually increased. Advances in surgical technique are inseparable with modern science and technology. We bind repair techniques to spine biomechanical principles and molecular biology theory. Pre-assessment and program design are conducted before surgery. We bring a new idea of bilateral pedicle screw and step tight closure for gradual shortening. Now, we reported kyphosis correction using posterior total spinal osteotomy and step orthopedic treatment in recent 8 years in the Department of Orthopedics of our hospital.

\section{Subjects and Methods}

Design: A retrospective case analysis.

Time and setting: Experiments were performed in the Department of Orthopedics of the 306 Hospital of Chinese PLA from March 2006 to March 2014.

Subjects: A total of 90 patients with severe angular kyphosis, 37 males and 53 females, with an average age of 47 years (range from 33 to 59 years). All patients were treated with posterior total spinal osteotomy and bilateral pedicle screw and step tight closure for gradual shortening and orthopedic fixation. There were 51 cases of old tuberculous angular kyphosis, 22 cases of traumatic kyphosis, and 17 cases of congenital kyphosis. The protocol was complied with the Declaration of Helsinki, and was approved by the Ethics Committee of the 306 Hospital of Chinese PLA in China. This study contained 90 patients and it should gain the consent from ethics committee or the patients themselves. General data are listed in Table 1.

Diagnostic criteria: Angular kyphosis is severe kyphosis deformity induced by various reasons, such as old tuberculosis, spinal fractures and congenital spinal deformity. Degenerative changes based on severe angular kyphosis of the spine will aggravate spinal cord injury and early degeneration. In severe cases, there will be incomplete paralysis of both lower extremities, or even complete paralysis.

\section{Table 1. General data of patients.}

\begin{tabular}{cc}
\hline Item & Value \\
\hline Gender (male: female) & $37: 53$ \\
Age (year) & $33-59(47)$ \\
Preoperative lordosis & $31^{\circ}-138^{\circ}\left(90.1^{\circ}\right)$ \\
\hline
\end{tabular}


Inclusion criteria: (1) Severe rigid thoracolumbar angular kyphosis; (2) with or without lower extremity neurological symptoms, complete paralysis or incomplete paralysis; (3) preoperative examination to exclude cardiovascular disease, serology laboratory in the normal range, negative C-reactive protein; (4) patients signed informed consent for treatment and testing program.

Exclusion criteria: Serious cardiovascular and cerebrovascular disease, severe infection, other severe metabolic diseases, cancer, metastatic cancer; diagnosis of the patients is not clear; ankylosing spondylitis patients combined with severe osteoporosis.

\section{Materials}

Our team used titanium alloy materials. Whether imported or domestically produced, the material itself is the same. The only difference is processing technology. Moreover, the processing technology in China has little difference from the imported products. More importantly, there is a strong interaction between magnetic field and stainless steel. Stainless steel can interfere with the magnetic signal. In a strong magnetic field, the stainless steel is also very easy to move.

The hardness of titanium alloy is harder than aluminum and magnesium, which are relatively soft. The hardness and strength of titanium alloy greatly increase after forming alloys, and its density is small and close to modulus of elasticity of bone. Titanium alloy is appropriate to physiological environment of human body. Metal dissolution will not appear during fixation. Titanium alloy has high strength, small density, good mechanical behavior, toughness and corrosion resistance. Ti-6Al-4V (TC4) and Ti-5Al-2.5Sn (TA7) are widely used titanium alloy at present.

\section{Methods}

Total spinal osteotomy: patients lied in prone position after anesthesia. A posterior median incision was made on the spinal column to expose bone structure taking kyphosis apex as a center. In accordance with Weinstein's method, 6 - 8 pairs of pedicle screws were implanted in proximal and distal ends of fusion segments on both sides of the vertebral body. Total spinal osteotomy was conducted in the kyphosis apex and entire vertebral body was resected. Length and curvature of the convex side on the fusion segments were measured using a magic wand. Pre-bending of the connecting rod was carried out according to above curvature. The connecting rod was placed on the convex side. Screw nuts were installed on the tail ends of the screws to fix the connecting rod and to protect the exposed spinal cord. Moreover, the length of the spinal cord was not changed (Figures 1(a)-(f)).

Bilateral pedicle screw and step tight closure for gradual shortening: after successful osteotomy, length of fusion segment and curvature of the concave side were measured with the magic wand. Pre-bending of the connecting rod was carried out according to above curvature. Temporary fixed rod on the concave side was removed, and the connecting rod was placed on the concave side. Screw nuts were installed on the tail ends of the screws to fix the connecting rod and to protect the exposed spinal cord. Moreover, the length of the spinal cord was not changed.

During orthopedics on the concave side, the fixed rod on the convex side was removed. Two locking pliers were used to fix orthopedic rod on the concave side near to both sides of osteotomy site. After locking nail cap, in situ orthopedics was conducted. Kyphosis was apparently improved under direct vision. Length of fusion segment and curvature of the convex side were measured with the magic wand. Pre-bending of the connecting rod was carried out according to above curvature. The connecting rod was placed on the convex side. Two bone holding forceps were utilized to hold the distal and proximal ends of osteotomy site. The nut was loosed at the proximal and distal ends. Thus, the screws were slowly moved on the connecting rod, resulting in slow pressure and reduction under direct vision. After scoliosis was partially improved, the nuts on the proximal and distal ends of the osteotomy site were tightened. The scoliosis was further improved under direct vision.

Orthopedics was repeated by the same method. Kyphosis was gradually corrected. The pre-bending degree of the orthopedic rod gradually reduced. Kyphosis was noticeably corrected. Under direct vision, dura mater that became thin due to oppression by bone structure gradually became short and thick. Fluctuations appeared on dural surface. The tail end of a nail was locked. Under direct vision, kyphosis and spine coronal shift were corrected. Subsequently, laminectomy and decompression were conducted in upper and lower vertebrae on the osteotomy plane. Using a nerve dissector, no evident compression was observed on dural sac and nerve root. No obvious active bleeding was visible within the surgical wound. A transverse connector was installed on the 


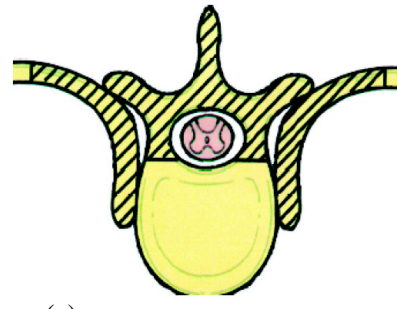

(a)

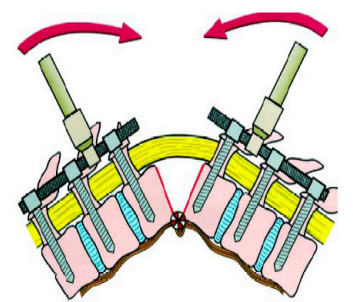

(d)

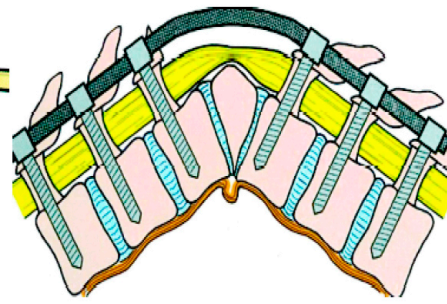

(b)

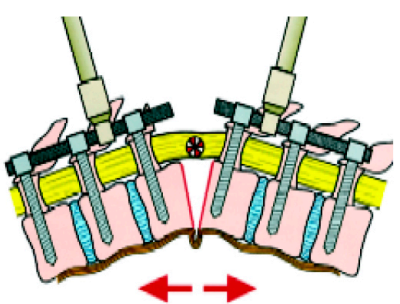

(e)

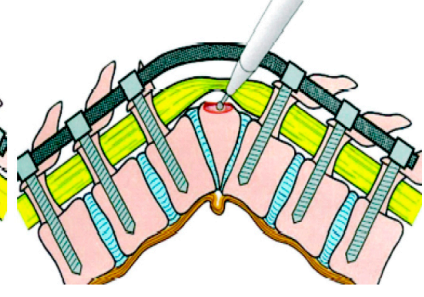

(c)

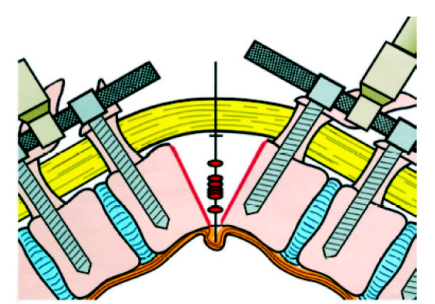

(f)

Figure 1. Gradual spinal shortening using bilateral pedicle screw and step tight closure. (a): Osteotomy site; (b): Exposure of dura mater after biting osteotomy site; (c): Temporarily fixed metal rod at osteotomy site to ensure the stability of the vertebral column. (a)-(c): Hinge center of step orthopedics located in the anterior longitudinal ligament; (d)-(f): Hinge center moved from anterior longitudinal ligament to posterior longitudinal ligament. Front began to open.

proximal and distal ends of osteotomy plane. During surgery, spinal cord shortened at most 2 cm. Changes in neurological function were monitored by using intraoperative wake-up test and somatosensory evoked potentials. Intraoperative wake-up test could identify the damage to the spinal cord. Thus, orthopedics could be performed according to the tolerance of spinal cord to surgical stretch, which effectively avoided the occurrence of intraoperative nerve injury (Figure 2).

Posterior screw rod fixation-moderate orthopedics + anterior titanium mesh support: after orthopedics was satisfactory, a fixed rod with suitable length was fixed on the concave side. The length of the anterior column defect was measured through broken ends of the osteotomy site on the convex side. A titanium mesh of suitable length was cut. Adequate autogenous bone was placed in the titanium mesh. After vertebral resection, the interspace was filled with titanium cage full of autologous bone + allogeneic bone to support graft. Under fluoroscopy, titanium mesh showed right size and satisfactory location. Appropriate pressure was given on the osteotomy site to ensure the stability of titanium mesh. The fixed rod of suitable length was selected for fixation on the convex side.

The Ti-5Al-2.5Sn (TA7) which was used in this study internal fixation of screw-rod system for titanium alloy materials, check in the future (including MRI) has no effect.

Main outcome measures: Preoperative and postoperative kyphotic angle, spinal sagittal imbalance, rate of trunk lateral displacement, operation time, intraoperative blood loss, Visual Analog Scale, Japanese Orthopaedic Association score, Oswestry Disability Index score and SF-36 score were analyzed in this study.

Statistical analysis: Data were analyzed using SPSS 13.0 software. The changes in preoperative and postoperative data were analyzed using $t$-test. A value of $\mathrm{P}<0.05$ was considered statistically significant.

\section{Results}

\subsection{Quantitative Analysis of Participants}

A total of 90 patients with severe angular kyphosis after treatment with posterior total spinal osteotomy and bilateral pedicle screw and step tight closure for gradual shortening and orthopedic fixation were included in final analysis, finished follow-up, no drop out.

\subsection{Intraoperative and Postoperative Conditions}

All patients received general anesthesia. Average operation time was 326 minutes (212 - 470 minutes). Average 

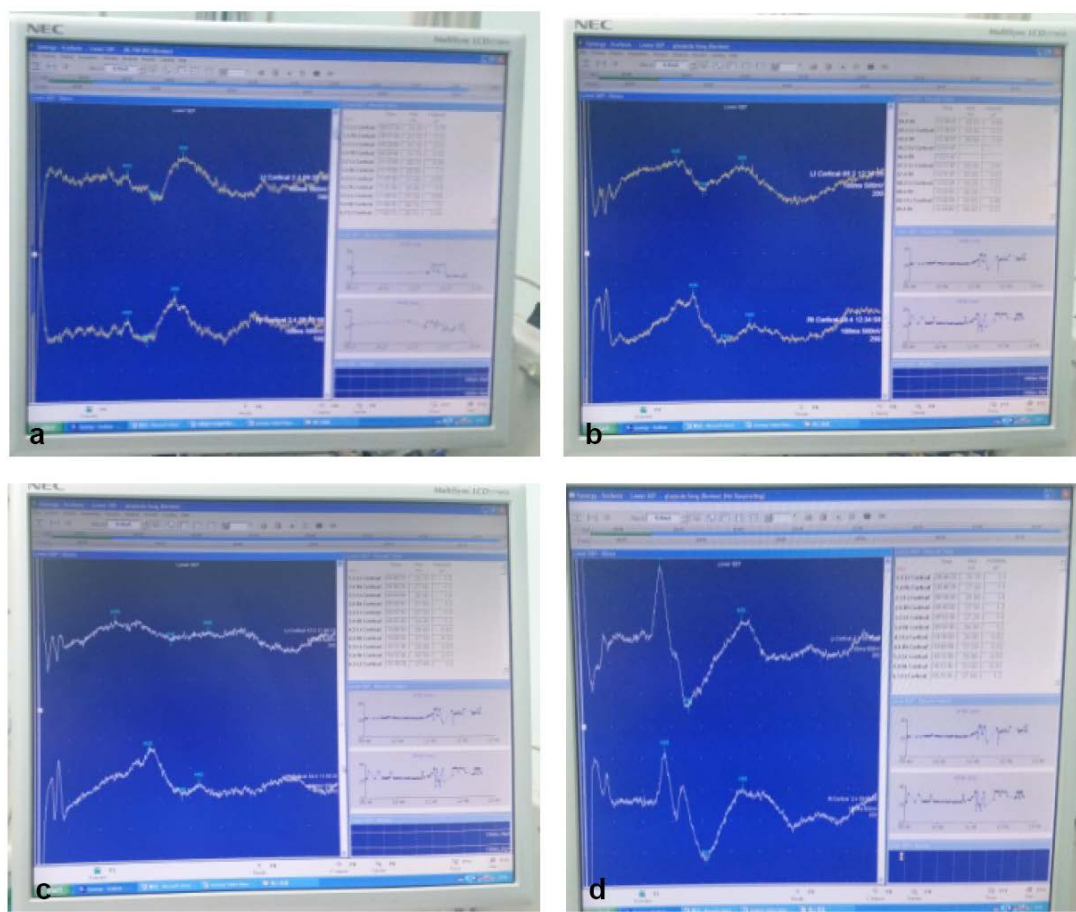

Figure 2. PTN-SEP wave form. Note: (a): PTN-SEP waveform preoperatively revealed that waveform in the left limb was smaller than that of the right limb, and the latency of wave crest prolonged; (b): During decompression, the amplitude of cortical potential of PTN-SEP waveform apparently reduced, and the latency of wave crest prolonged (amplitude $0.59 \mu \mathrm{v}$; P28 latency $47.66 \mathrm{~ms}$ ); (c): Cauda equina and cortical potential amplitude even disappeared at one time; (d): PTN-SEP waveform after total vertebral osteotomy and spinal decompression becomes normal.

intraoperative blood loss was $2089 \mathrm{~mL}(1200$ - $6000 \mathrm{~mL}) .104$ vertebral bodies were resected during surgery. There were 58 cases of single corpectomy, 20 patients receiving corpectomy of top vertebra of major curve and top vertebra of compensatory curve, and 2 patients undergoing corpectomy of top vertebra of major curve, adjacent vertebral bodies and top vertebra of compensatory curve. Osteotomy plane was at $\mathrm{T}_{4}-\mathrm{L}_{2}$. Follow-up was conducted for 20 to 35 months.

Management of intraoperative and postoperative complication: during spinal cord monitoring, orthopedic angle was decreased in the patients whose somatosensory evoked potential reduced. Moreover, osteotomy site was explored and the scope of decompression was expanded. After intraoperative hormone therapy, neurological symptoms of lower extremities were restored in five patients. Of them, two patients received secondary surgical exploration (Table 2).

\subsection{Follow-Up Results}

Average kyphotic angle was $41.6^{\circ}\left(10^{\circ}-90^{\circ}\right)$ with a correction rate of $65 \%$. The distance from $\mathrm{C}_{7}$ plumb line to posterior upper edge of the $S_{1}$ vertebral body was averagely $5.2 \mathrm{~mm}$ (-12 to $23 \mathrm{~mm}$ ) postoperatively, with a correction rate of $73 \%$.

\subsection{Typical Case}

A 29-year-old male patient with severe rigid kyphosis underwent posterior total spinal osteotomy and bilateral pedicle screw and step tight closure for gradual shortening under general anesthesia. Kyphotic angle changed from $165^{\circ}$ preoperatively to $50^{\circ}$ at 1 year after operation, with a correction rate of $70 \%$ (Figure 3, Figure 4).

\subsection{Adverse Events}

Neurological complications occurred in 5 cases, accounting for 6\%, including 1 case of postoperative hematoma compression, 1 case of residual bone oppression at osteotomy site, 2 cases of displacement of osteotomy site, 
Table 2. Intraoperative and postoperative conditions of patients.

\begin{tabular}{ll}
\hline Item & Observational data \\
\hline Operation time (min) & $212-470(326)$ \\
Intraoperative blood loss (mL) & $1200-6000(2,089)$ \\
Osteotomy plane & $\mathrm{T}_{4}-\mathrm{L}_{2}$ \\
Number of resected vertebra $(n)$ & \\
Single vertebra & 57 \\
Two segments & 20 \\
Follow-up (mon) & $20-35(28.0)$ \\
Correction angle of kyphosis $\left({ }^{\circ}\right)$ & $10-90(41.6)$ \\
Correction of trunk shift $\left({ }^{\circ}\right)$ & $12-23(5.2)$ \\
Improvement rate of Visual Analog Scale & $83 \%$ \\
Improvement rate of Japanese Orthopaedic Association score & $82 \%$ \\
Oswestry disability index score & $19.13 \pm 67$ \\
SF-36 score & $337.69 \pm 86.17$ \\
Complications & \\
Nervous system in 5 cases (6\%) & Hematoma compression in 1 case, Inadequate decompression in 1 case, \\
displacement of osteotomy site in 2 cases, and nerve root irritation in 1 \\
Non-nervous system in 5 cases (6\%) \\
\hline
\end{tabular}
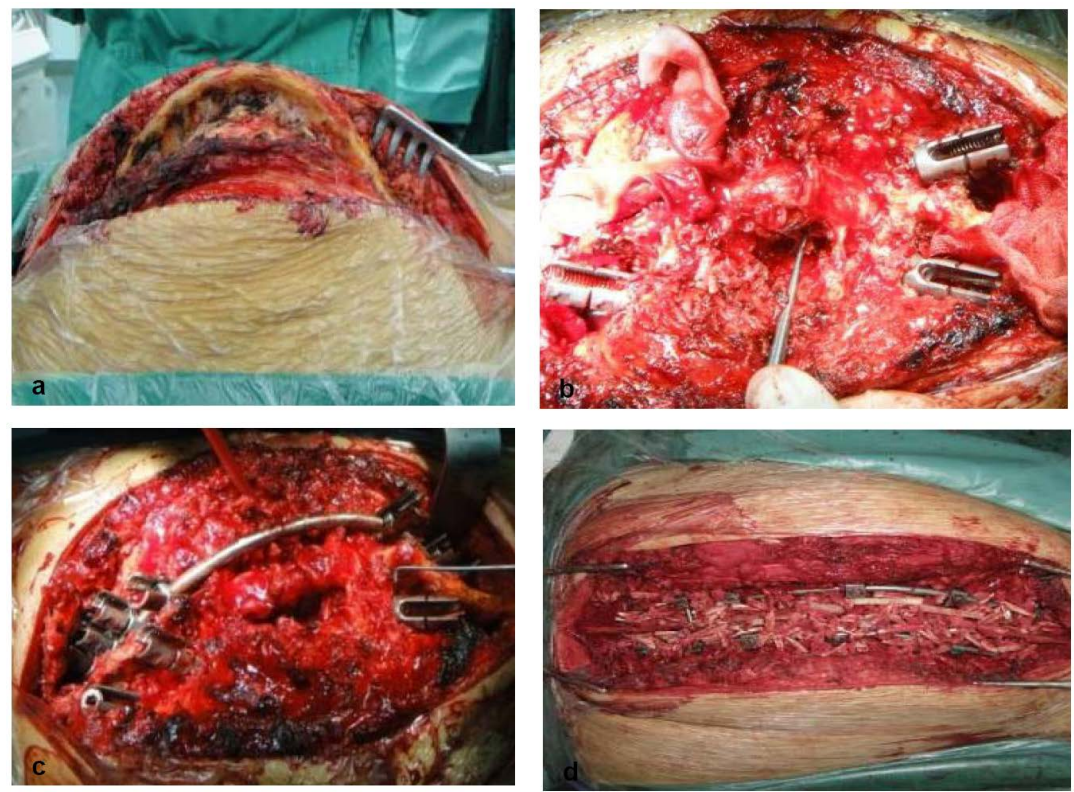

Figure 3. Application of bilateral pedicle screw and step tight closure for gradual shortening. Note: (a): Osteotomy site of angular kyphosis; (b): After total spinal osteotomy; (c): During step shortening; (d): After osteotomy with internal fixation.

and 1 case of nerve root irritation. Non-neurological complications occurred in 5 cases, accounting for 6\%, including 3 cases of pleural effusion, 1 case of wound infection, and 1 case of lose of orthopedic angle. Three patients with pleural effusion were subjected to thoracentesis. Their symptoms lessened after closed-chest drainage. One patient with wound infection was subjected to debridement, and the wound was healed. During 2-year follow-up, the connecting rod of one patient broke, and orthopedic angle lost. This patient received reoperation to replace the connecting rod. 

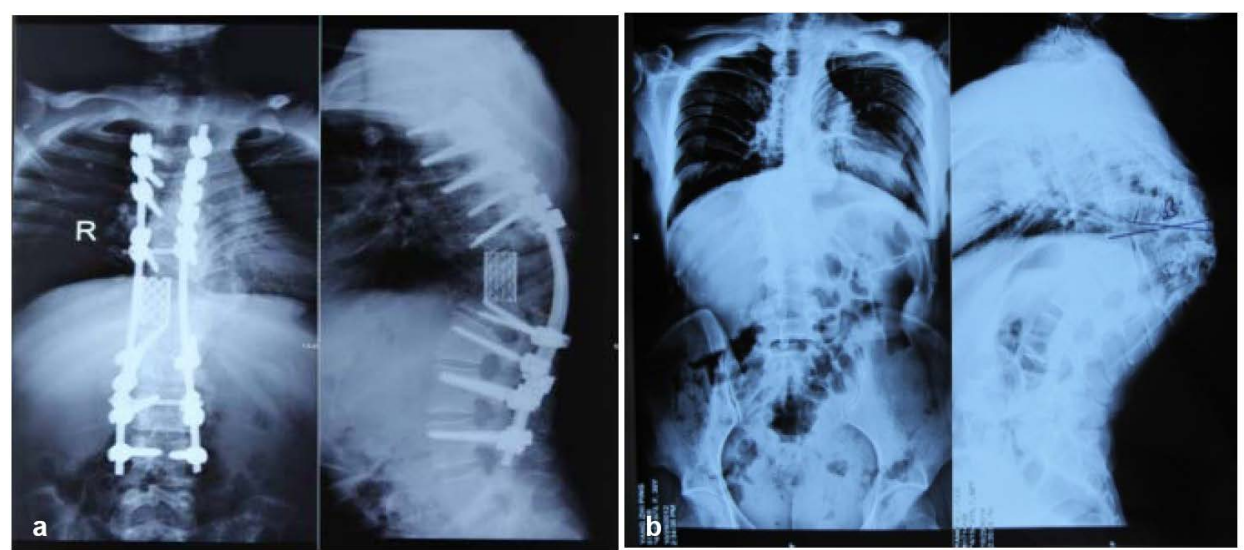

Figure 4. Anteroposterior and lateral radiographs of the whole spine in a patient with severe rigid kyphosis before and after posterior total spinal osteotomy and step orthopedic treatment for gradual shortening. Note: (a): Anteroposterior and lateral radiographs of the whole spine before operation, kyphotic angle was $165^{\circ}$; (b): Anteroposterior and lateral radiographs at 1 year after surgery, kyphotic angle was $50^{\circ}$, with the correction rate of $70 \%$.

During spinal cord monitoring, orthopedic angle was decreased in the patients whose somatosensory evoked potential reduced. Moreover, osteotomy site was explored and the scope of decompression was expanded. After intraoperative hormone therapy, symptoms relieved. Neurological symptoms of lower extremities were restored in five patients. Of them, two patients received secondary surgical exploration.

\section{Discussion}

Previous studies confirmed that bone of angular kyphosis patients at deformity plane was associated with congenital defect of spinal vascular damage and the compression of damaged spine structure on corresponding segments of the spinal cord [3] [4]. Spinal cord blood system disorders and structural spinal cord compression syndrome cause destruction of spinal cord function, which are reasons for neurological symptoms of spinal cord during and after corrective surgery of angular kyphosis. If the patient affected neurological symptoms before surgery, the risk of aggravated neurological symptoms is great after surgery. Simultaneously, biomechanical feature of soft tissues when subjected to stress is the occurrence of hysteresis, relaxation and creep of stressstrain curve [5]. In initial stage, stress increases rapidly, but becomes slow gradually. When displacement load is stable, viscoelastic cells present stress relaxation, but linear stress of cells remains unchanged. If the diameter of the nucleus is bigger, the position of maximum stress point is closer to the cell edge, and the maximum stress is greater. Therefore, according to biomechanical variation after cells sustain forces, this study used step tight closure for gradual shortening at osteotomy site. We guaranteed fluid intake and added the necessary energy during surgery. Slow drag during surgery effectively guarantees surgical safety.

Moreover, the combined application of pedicle screw fixation can effectively prevent lateral displacement at distal osteotomy site. After osteotomy, shrinkage occurs in traction-induced tapered spinal cord during shortening. To prevent spinal cord injury and nerve entrapment, decompression should be conducted in proximal and distal ends of osteotomized segment during osteotomy. For patients with spinal nerve disorders before surgery, adhesive nerve root and dural sac should be carefully peeled off during the surgery so as to reduce the probability of spinal nerve injury, which effectively avoids the occurrence of complications and the surgical procedures will be safer and effective. These results are not mentioned in previous studies [6]-[8].

Ideal bone graft should be characterized by bone formation, bone induction and bone conduction [9] [10], can provide certain shape and structure, and withstand a certain load. We used autologous bone material with capacity of bone induction, without rejection, and the autologous bone material does not spread disease. The fixator used in this study is made of titanium alloy, which cannot impact future examination (including MRI).

Numerous previous studies demonstrated that the difficulty of angular kyphosis surgery lies in low correction rate, difficult to reconstruct sagittal plane, easy to cause neurological complications, postoperative imbalance, high incidence rate of pseudarthrosis, and loss of postoperative orthopedic angle [11]-[21]. In this study, $360^{\circ}$ decompression was conducted in top spinal vertebrae during posterior total spinal osteotomy. Temporary fixa- 
tion was used on the first completed side to prevent distal displacement. In the combination of our clinical observation, methods for avoiding the complications of nerve injury mainly contain accurate placement of pedicle screw, fixation with temporary rod during osteotomy, fixation with screws in upper and lower vertebrae of osteotomy site to reduce displacement, adequate scope of decompression, avoiding incomplete osteotomy or residual bone in front of the spinal canal, careful operation to avoid devices-induced accidental injury, timely shortening after decompression so as to avoid spinal cord injury, maintaining unobstructed drainage and avoiding hematoma after surgery.

The advantages of the study introduced technique in the treatment of angular kyphosis are: this research section joints proximal and after internal fixation with pedicle screws to prevent lateral displacement of distal osteotomy. Osteotomy with spinal shortening after the completion of the process, along with osteotomy and ends near each other, stretching thin spinal shrinkage during the process of shortening occurs, resulting in spinal injuries and nerve compression, osteotomy should be further cut bone in proximal and laminectomy. Meanwhile, during the PCR osteotomy orthopedic correction with spinal compression exploration in spine cord, this spinal cord compression can be prevented effectively, achieve the purpose of shortening the spinal cord, can improve the nerve function. Before spinal PCR osteotomy orthopedic correction, we need clear disabilities, and carefully adhesion of nerve root, reducing the probability of spinal cord or nerve injury. Avoiding the occurrence of complications, surgical procedures are safer and more effective. This is not mentioned in the previous reports.

No relevant reports are retrieved. That is to say, posterior total spinal osteotomy combined with bilateral pedicle screw and step tight closure for gradual shortening belongs to innovative technology in treatment of severe rigid angular kyphosis. In the present study, patients were followed up for 20 - 35 months (averagely 28 months). Average kyphotic angle was $41.6^{\circ}\left(10^{\circ}-90^{\circ}\right)$ with a correction rate of $65.3 \%$. The distance from $C_{7}$ plumb line to posterior upper edge of the $S_{1}$ vertebral body was averagely $5.2 \mathrm{~mm}$ (-12 to $23 \mathrm{~mm}$ ) postoperatively, with a correction rate of $73.0 \%$. Above data verified that our therapeutic method is safe and stable. However, the posterior total spinal osteotomy for treating severe rigid angular kyphosis showed great trauma and more complications. The surgical technique is difficult and critical, demands a long time to complete the learning curve.

With the development of anterior and posterior spinal osteotomy spine reconstruction in recent years, the scoliosis kyphosis provides new methods of treatment. During operation, it should tie in with the spinal cord monitoring to avoid nerve damage occurrence in order to ensure the operation safety.

Implementation posterior thoracolumbar osteotomy with internal fixation can effectively correct kyphoscoliosis to obtain a satisfactory spinal sagittal and coronal balance.

\section{References}

[1] Ji, J.G., Zhang, X., Qiu, G.X., Xing, Z.J., Wang, Y.P., Shen, J.Q., Zhao, Y. and Li, S.G. (2010) Selection of the Lowest Instrumented Vertebrae for Adolescent Idiopathic Scoliosis. Chinese Journal of Orthopaedics, 30, 321-324.

[2] Lu, M., Fischenko, V.Y., Vleschenko, V.A. and Fischenko, Y.V. (2010) Etiological Research on Structural Features of Paraspinal Muscles in Patients with Scoliosis: In Molecular Biology Level. Clinical Medical Engineering, 17, 1-3.

[3] Lu, M., Fischenko, V.Y., Vleschenko, V.A. and Fischenko, Y.V. (2010) Studies on Monomer Radionuclide Diffraction of Paraspinal Muscles and Observation on Biochemical Characteristics of Muscle Shortening Protein in Scoliosis Patients. Clinical Medical Engineering, 17, 1-4.

[4] Lu, M., Fischenko, V.Y., Vleschenko, V.A. and Fischenko, Y.V. (2010) Structural Characteristics of Nucleus Pulposus and Related Etiology in Patients with Idiopathic Scoloosis. Chinese Journal of Tissue Engineering Research, 14, 7008.

[5] Sun, Y.S., Liang, C. and Wen, J.M. (2011) Lumbar Interbody Fusion and Clinical Application. Chinese Journal of Clinicians, 5, 4621-4626.

[6] Yan, L.Q., Huan, C., Sun, Y., Wang, J.C., Liu, R., Zhang, W.L., Yu, T.Y., Feng, X.M., Tao, Y.P. and Yang, J.D. (2011) Biomechanical Evaluation and Comparison of Isola Pedicle Screw Internal Fixation to DIAM Fixation for Lumbar Spine. Chinese Journal of Clinicians, 5, 4432-4437.

[7] Shi, Y., Chang, C., Yang, L., Jiang, Z.W., Wang, G.D. and Shi, B.H. (2011) Clinical Outcomes of Posterior Lumbar Interbody Fusion versus Posterolateral Fusion in Lumbar Degenerative Disease. Chinese Journal of Clinicians, 5, 2394-2398.

[8] Wang, Y., Zhang, Y.G., Zheng, G.Q., Xiao, S.H., Zhang, X.S. and Wang, Z. (2010) Vertebral Column Decancellation for the Management of Rigid Scoliosis: The Effectiveness and Safety Analysis. Chinese Journal of Surgery, 48, 1701-1704.

[9] Fan, J.P., Wang, C.F., Zhu, X.D., Li, C., Chen, C., Yi, H.L., Yang, C.W., Bai, Y.S. and Li, M. (2013) Clinical Analysis 
of Surgical Treatment for Adult Idiopathic Scoliosis with Ponte Osteotomy. Journal of Clinical Orthopaedics, 16, 121-124.

[10] Hamzaoglu, A., Alanay, A., Ozturk, C., Sarier, M., Karadereler, S. and Ganiyusufoglu, K. (2011) Posterior Vertebral Column Resection in Severe Spinal Deformities: A Total 102 Cases. Spine (Phla Pa 1976), 36, E340-E344.

[11] Yao, Z.M., Zhang, J.G., Qiu, G.X., Wang, S.R., Weng, X.S. and Guo, J.W. (2013) Perioperative Complications and Risk Factors of One-Stage Posterior Vertebral Column Resection for Severe Spinal Deformity. Chinese Journal of Orthopaedics, 33, 440-446.

[12] Lenke, L.G., Sides, B.A., Koester, L.A., Hensley, M. and Blanke, K.M. (2010) Vertebral Column Resection for the Treatment of Severe Spinal Deformity. Clinical Orthopaedics and Related Research, 468, 687-699. http://dx.doi.org/10.1007/s11999-009-1037-x

[13] Lenke, L.G., O’Leary, P.T., Bridwell, K.H., Sides, B.A., Koester, L.A. and Blanke, K.M. (2009) Posterior Vertebral Column Resection for Severe Pediatric Deformity Minimum Two Year Follow-Up of Thirty-Five Consecutive Patients. Spine (Phila Pa 1976), 34, 2213-2221. http://dx.doi.org/10.1097/BRS.0b013e3181b53cba

[14] Xie, J., Wang, Y., Zhao, Z., Zhang, Y., Si, Y., Li, T., Yang, Z. and Liu, L. (2012) Posterior Vertebral Column Resection for Correction of Rigid Spinal Deformity Curves Greater than $100^{\circ}$. Journal of Neurosurgery: Spine, 17, 540-551. http://dx.doi.org/10.3171/2012.9.SPINE111026

[15] Lenke, L.G., Newton, P.O., Sucato, D.J., Shufflebarger, H.L., Emans, J.B., Sponseller, P.D., Shah, S.A., Sides, B.A. and Blanke, K.M. (2013) Complications after 147 Consecutive Vertebral Column Resections for Severe Pediatric Spinal Deformity: A Multicenter Analysis. Spine (Phila Pa 1976), 38, 119-132. http://dx.doi.org/10.1097/BRS.0b013e318269fab1

[16] Ma, H.S., Chen, Z.M., Yang, B., Wu, J.G., Tan, R. and Wang, X.P. (2012) Analysis of Neurological Deficits Complications in the Treatment of Spinal Deformity with Posterior Spinal Osteotomy. Chinese Journal of Surgery, 50, 328-332.

[17] Schairer, W.W., Carrer, A., Lu, M. and Hu, S.S. (2014) The Increased Prevalence of Cervical Spondylosis in Patients with Adult Thoracolumbar Spinal Deformity. Journal of Spinal Disorders \& Techniques, 27, E305-E308. http://dx.doi.org/10.1097/BSD.0000000000000119

[18] Kanter, A.S., Shaffrey, C.I., Mummaneni, P., Wang, M.Y. and Uribe, J.S. (2014) Introduction: Adult Spinal Deformity: Pathophysiology and Corrective Measures. Neurosurgical Focus, 36.

[19] Fujimori, T., Inoue, S., Le, H., Schairer, W.W., Berven, S.H., Tay, B.K., Deviren, V., Burch, S., Iwasaki, M. and Hu, S.S. (2014) Long Fusion from Sacrum to Thoracic Spine for Adult Spinal Deformity with Sagittal Imbalance: Upper versus Lower Thoracic Spine as Site of Upper Instrumented Vertebra. Neurosurgical Focus, 36, E9. http://dx.doi.org/10.3171/2014.3.FOCUS13541

[20] Fu, J., Zhang, G., Zhang, Y., Liu, C., Zheng, G., Song, K., Tang, X., Zhang, X. and Wang, Y. (2014) Pulmonary Function Improvement in Patients With Ankylosing Spondylitis Kyphosis Following Pedicle Subtraction Osteotomy. Spine (Phila Pa 1976), 39, E1116-E1122. http://dx.doi.org/10.1097/BRS.0000000000000441

[21] Clement, J.L., Chau, E., Geoffray, A. and Suisse, G. (2014) Restoration of Thoracic Kyphosis by Simultaneous Translation on Two Rods for Adolescent Idiopathic Scoliosis. European Spine Journal, 23, S438-S445. http://dx.doi.org/10.1007/s00586-014-3340-9 